\title{
Simulation of Cosmonaut Rescue Using Virtual Reality
}

\author{
Mikhail Mikhaylyuk ${ }^{1}$, Andrey Maltsev ${ }^{1}$ and Evgeny Strashnov ${ }^{1}$ \\ ${ }^{1}$ Federal State Institution «Scientific Research Institute for System Analysis of the Russian Academy of Sciences», \\ 36-1 Nakhimovskiy Ave., Moscow, 117218, Russia
}

\begin{abstract}
This paper presents original solutions for creation of training complex learning cosmonauts to control a space jet pack on purpose self-rescue when emergency happens. An approach is proposed in which training is carried out in a virtual environment using virtual reality gloves and headset. The idea is that control of virtual space jet pack model is performed by interaction of virtual hands, copying movements of cosmonaut's hands, with three-dimensional model of jet pack's control panel. To implement the training complex, methods and approaches were developed for movement synchronization simulation of virtual and real hands, as well as simulation of jet pack's control panel and thrusters. Approbation of proposed methods and approaches was carried out as part of our virtual environment system VirSim developed at the SRISA RAS. Results obtained in the paper can be used to create training complex for learning cosmonauts to rescue when they accidentally separate from the International Space Station.
\end{abstract}

\section{Keywords}

Virtual reality, simulation, stereo visualization, training complex, space jet pack, virtual control panel, VR headset.

\section{Introduction}

Currently, cosmonauts periodically need to carry out spacewalks and perform operations on the surface of the International Space Station (ISS). Special safety tethers are actively used for such operations, with the help of which cosmonaut attaches himself to spacecraft. However, there is a risk of unsuccessful attachment or malfunction of the tether, with the result that cosmonaut is detached from spacecraft and cannot return. In the event of this emergency, it is relevant to use cosmonaut rescue system (for example, SAFER - Simplified Aid For EVA Rescue [1, 2]), which represents a space jet pack put on spacesuit. The pack includes a set of thrusters, which create a thrust by means of supplying compressed air from a balloon. It allows cosmonaut to implement movements and rotations in weightlessness. Special control panel is used to control space jet pack in manual or automatic mode. The problem is to teach cosmonauts the skills of such control when training in terrestrial conditions. Therefore, an important and topical area of research is the creation of training systems for learning cosmonauts to use rescue equipment when separation from the ISS.

In recent years, advanced technologies of virtual reality (VR) are actively introduced into various spheres of human life. In particular, space simulators $[3,4,5]$ are created which train cosmonaut by means of immersion in virtual environment. The advantage of VR technology application is in improving of visual perception quality of computer-synthesized environment by the operator. Many scientific groups are engaged in development of such space simulators. Motion tracking systems for parts of a person's body (head, torso, legs and hands) were considered in publication [3] on the example of cosmonaut performing various operations during a spacewalk. Paper [4] presents a developed simulator for controlling manned spacecraft by joysticks as part of the planned mission of the Moon exploration. Hardware solutions [5] based on VR technologies and created in the NASA laboratory are

GraphiCon 2021: 31st International Conference on Computer Graphics and Vision, September 27-30, 2021, Nizhny Novgorod, Russia EMAIL: mix@niisi.ras.ru; avmaltcev@mail.ru; strashnov_evg@mail.ru ORCID: 0000-0002-7793-080X; 0000-0003-1776-814X; 0000-0003-0937-4052

(c) (i) 2021 Copyright for this paper by its authors.

Use permitted under Creative Commons License Attribution 4.0 International (CC BY 4.0).

CEUR Workshop Proceedings (CEUR-WS.org) 
widely used. They also include training cosmonauts to control space jet pack with using a prototype of real control panel.

This paper presents new solutions for implementation of training complex that learns cosmonauts to control space jet pack. They are based on VR technologies and our virtual environment system. The main scientific idea is that a control of jet pack model is executed by interaction of real cosmonaut with elements of virtual three-dimensional control panel. For this, the Oculus Rift CV1 virtual reality headset, Oculus Touch controllers and Manus Prime II VR gloves are used as hardware solutions. These devices allow implementing visual feedback with virtual environment and tracking motions of operator's head and hands. Suggested solutions include methods and approaches for control and dynamics simulation of virtual hands, elements of virtual control panel and virtual model of cosmonaut with space jet pack. Approbation of proposed methods and approaches was carried out as part of our virtual environment system (VES) VirSim. It showed their effectiveness for learning control of space jet pack.

\section{Hardware and software of training complex}

Developed training complex of cosmonaut rescue consists of three major groups of components: hardware block, software complex and digital visual models (DVM). Figure 1 illustrates the structure proposed for this complex. Hardware block solves the problem of tracking the operator's head, hands and fingers, as well as transmission of virtual environment images to his eyes. Full tracking system for hands and fingers is implemented on the base of original union two independent devices: Manus Prime II VR gloves and Oculus Touch controllers (Figure 2). The latter are necessary to determine hand positions in real space due to the lack of such functionality in Manus gloves. Data received from sensors of Oculus Rift CV1 headset are used to track current orientation of head. Furthermore, the headset is need to display rendered image of virtual scene.

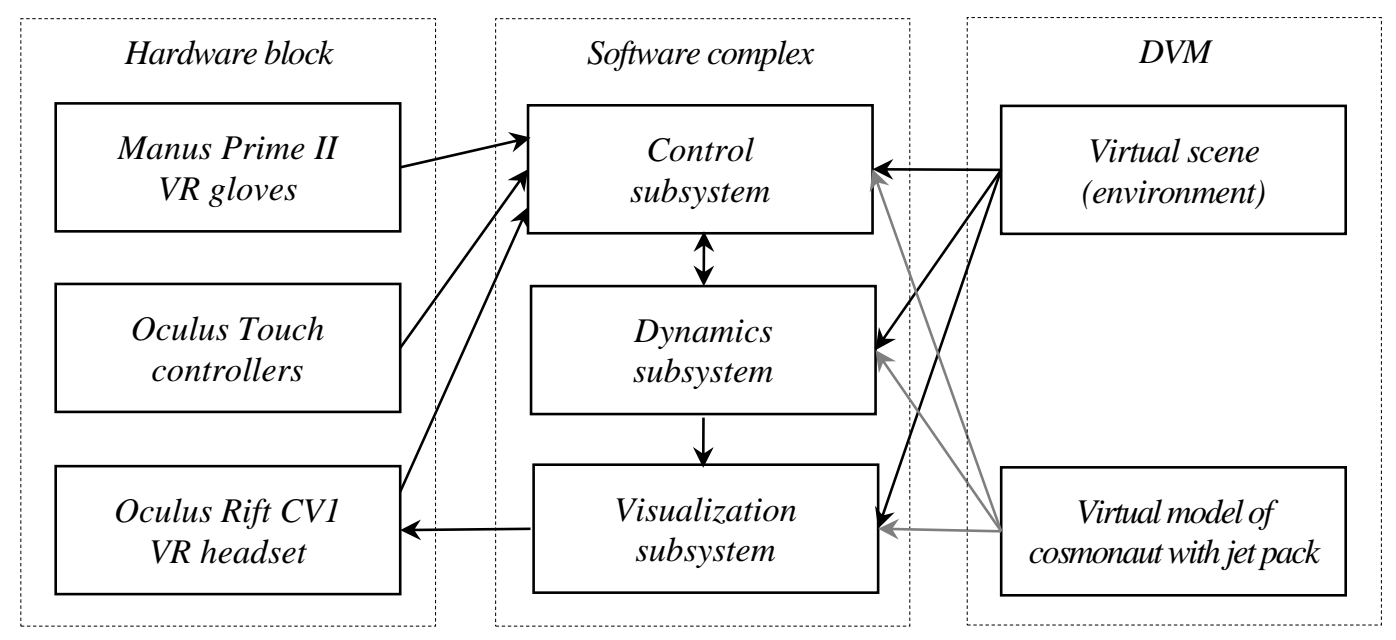

Figure 1: The structure of VR training complex for cosmonaut rescue simulation

Software complex consists of control, dynamics and visualization subsystems. They are original products, which were designed from the ground up without using third-party software. Control subsystem provides receiving information from hardware block, as well as virtual control elements located in DVM. Acquired data are used to compute control schemes [6] for dynamic elements of DVM and generate control signals on this basis with transmitting them to dynamics subsystem. Based on these signals, dynamics subsystem computes new positions and orientations of virtual scene's moving objects, after which it executes collision detection and response between objects in the scene. Visualization subsystem performs distributed rendering of virtual environment on multi-core graphics processor (GPU) with computing realistic illumination of its objects. The result of the rendering is a stereo pair, which is sent to the Rift VR headset. All components of the software complex provide realtime operation. 


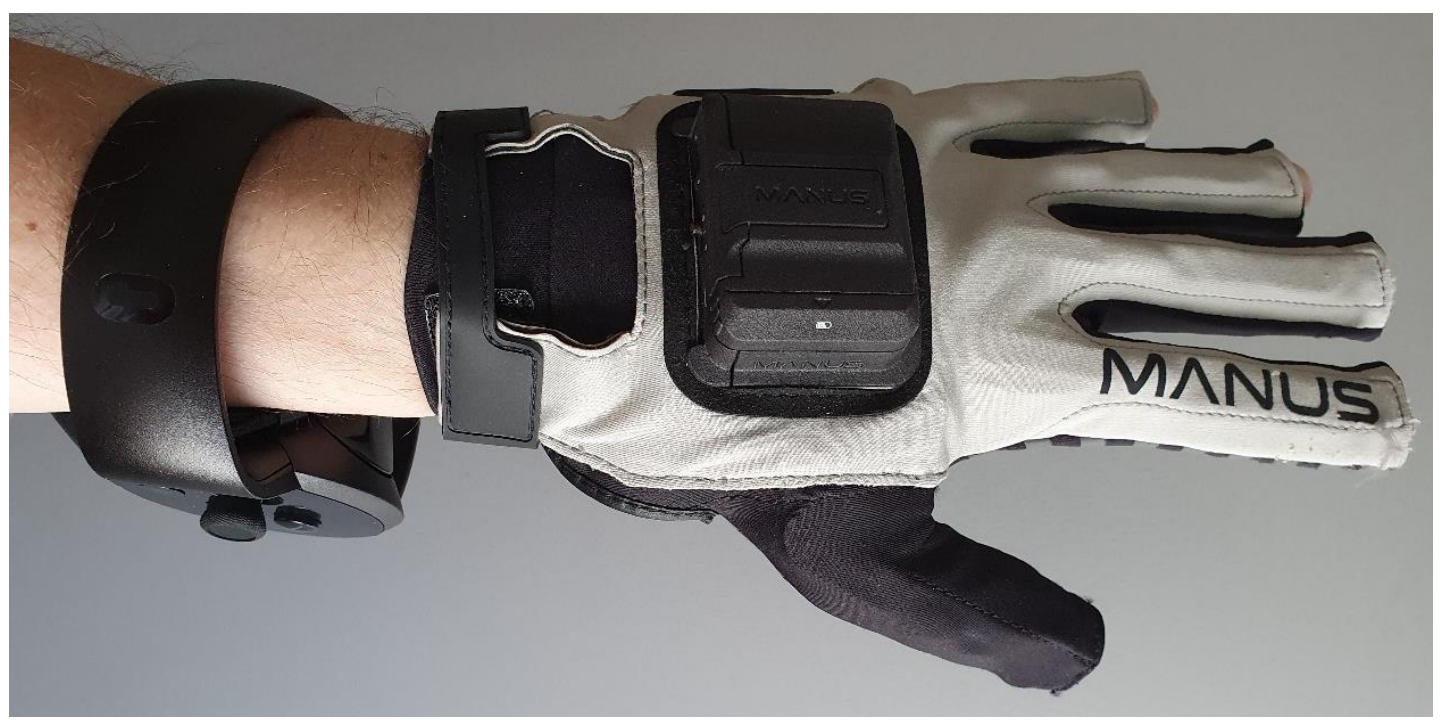

Figure 2: Union of Manus Prime II and Oculus Touch for tracking hand and fingers

DVM of training complex includes two part. The first is own highly detailed virtual scene of cosmonaut environment (models of the ISS and the Earth) containing about a million polygons. The second is virtual model of cosmonaut with jet pack created by us. In addition to geometry and textures, this model also contains functional scheme for control of its head rotation, hand and fingers movements based on signals from hardware block, as well as operation of rescue device's thrusters based on signals from toggle switches and joystick located on its virtual control panel. Next, we will consider in more detail methods and approaches of dynamics and control simulation for virtual model of cosmonaut with space jet pack.

\section{Simulation of virtual hand}

Self-rescue training for cosmonaut with jet pack consists in practicing operations, most of which are performed with the help of his hands. Therefore, to solve the assigned task, we created a virtual model of the hand shown in Figure 3. Its design was carried out in the computer simulation system 3ds Max. The compressions and stretches of the fabric upper layers in this model, resulting from the bending of the fingers, are set by applying special skin modifier to polygonal geometry. For this, developed model contains a set of bones, changing positions and orientations of which affects vertices of its geometry with some coefficients.

In addition to the bones and visual model of virtual hand, developed VES also uses its dynamic model. It is represented as a system of articulated bodies, which is described by a set of generalized coordinates $\mathbf{q}=\left(q_{1}, \ldots, q_{N}\right)^{\mathrm{T}}$, where $N$ is the number of degrees of freedom. These

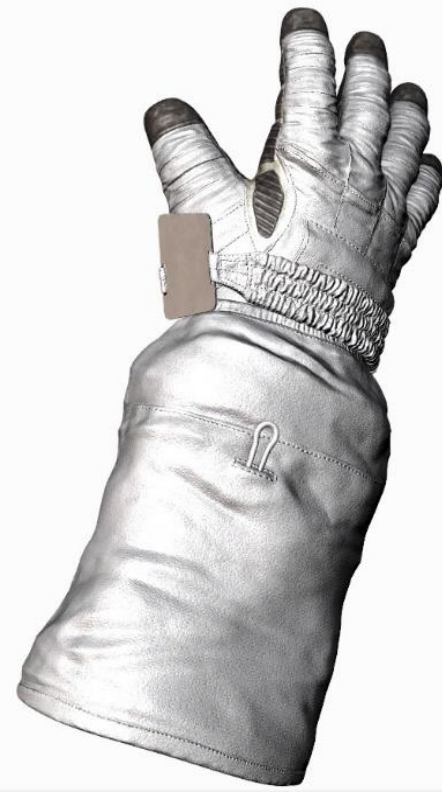

Figure 3: Virtual hand model coordinates define position and orientation of the hand, as well as joint angles of fingers. The task of hand dynamics simulation is to ensure its movement in zero gravity and in the presence of collisions with virtual environment objects. In general, collision response includes modeling contact, impact, and friction between interacting bodies. This leads to the fact that it is required to handle $M$ constraints 
imposed on the coordinates and velocities of bodies. It is known [7] that articulated bodies dynamics in the presence of constraints is described using the differential equations

$$
\mathbf{M}(\mathbf{q}) \ddot{\mathbf{q}}+\mathbf{C}(\mathbf{q}, \dot{\mathbf{q}}) \dot{\mathbf{q}}=\mathbf{Q}_{e}+\mathbf{J}^{T} \lambda,
$$

where $\mathbf{M}(\mathbf{q}) \in \mathbb{R}^{N \times N}$ is the mass matrix, $\mathbf{C}(\mathbf{q}, \dot{\mathbf{q}}) \in \mathbb{R}^{N \times N}$ is the Coriolis matrix, $\mathbf{Q}_{e} \in \mathbb{R}^{N}$ is the vector of applied forces (torques applied at hand's joints), $\mathbf{J} \in \mathbb{R}^{M \times N}$ is the constraint Jacobian matrix, $\lambda \in \mathbb{R}^{M}$ is the vector of Lagrange multipliers.

Simulation of the hand dynamics by formula (1) is implemented by means of extended version of the recursive Featherstone method, in which the constraint processing and computation of the vector $\lambda$ are based on the impulse approach [8] and integration of the equations of motion using the semi-implicit Euler scheme.

Virtual hand control is realized in copying mode by means of Manus VR glove and Oculus Touch controller, which are put on the human hand. Then the hand position and orientation, as well as joint angles of fingers, define the target vector $\mathbf{q}_{d}$ of generalized coordinates. Proposed solution for virtual hand control is that the torque $\mathbf{Q}_{e}$ is formed in the following form

$$
\mathbf{Q}_{e}=-\mathbf{K}_{p}\left(\mathbf{q}-\mathbf{q}_{d}\right)-\mathbf{K}_{d}\left(\dot{\mathbf{q}}-\dot{\mathbf{q}}_{d}\right),
$$

where $\mathbf{K}_{p}$ and $\mathbf{K}_{d}$ are diagonal matrices with positive coefficients.

Control law (2) allows realizing the movement of virtual hand in the copying mode.

\section{Simulation of virtual control panel for space jet pack}

In this paper, an approach is proposed in which control of virtual space jet pack model is realized using virtual three-dimensional control panel, the prototype of which is the hand controller module of the SAFER rescue system [1, 2]. Created model of this panel is shown in Figure 4. It contains a display, two status indicators, three toggle switches, one button and four-axis joystick with a button. The display is used to show remaining fuel and battery level in percent. Indicators of control panel status consist of two LEDs. The red LED labeled "THR" lights when the thrusters are active, and the green LED labeled "AAH" lights when the automatic attitude hold is enabled. Two-position toggle switch labeled "PWR" turns control panel on and off. Two-position toggle switch labeled "MODE" selects motion modes,

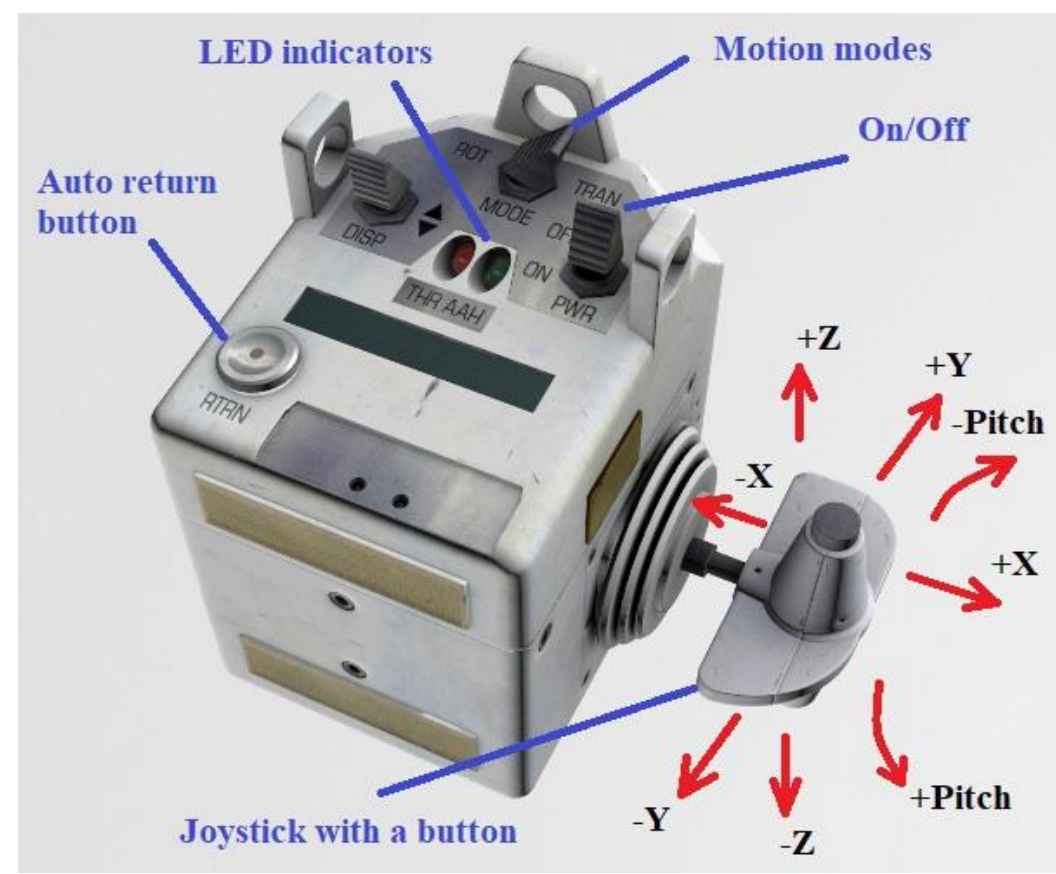

Figure 4: Virtual model of the space jetpack control panel 
where "ROT" and "TRAN" are associated with rotational and translational motion commands, respectively. The button labeled "RTRN" initiated mode of automatic return of cosmonaut to predetermined position. The joystick has three rotary axes and one transverse axis, which set movements and rotations of cosmonaut model. For example, deflection of the joystick in the Z-axis direction when the "ROT" mode is on sets rotation around the roll axis. The mode of automatic attitude hold for cosmonaut is switched on and off by means of the joystick's button.

Interaction of the operator with developed control panel is performed using created hand model. Controllable elements of the panel have a set of dynamic objects and sensors that compute the state of these elements. For toggle switches, rotation angles are determined, and for buttons - their displacements. The state of the joystick is determined by an offset along the $X$-axis and three successive rotations around the $Z, Y$, and $X$ axes. Coordinate values of virtual panel's elements are processed in control subsystem of our training complex and form commands to turn on and off the thrusters.

\section{Motion simulation of cosmonaut with jet pack}

The motion of cosmonaut in Earth's orbit is described relative to the ISS. Consider the world coordinate system (WCS) $O_{S} x y z$ and the local coordinate system (LCS) $O_{C} x^{\prime} y^{\prime} z^{\prime}$, which is rigidly attached to cosmonaut, as shown in Figure 5. Then cosmonaut position is defined with radius-vector $\mathbf{r}=(x, y, z)^{\mathrm{T}}$, and attitude - with transformation matrix $\mathbf{R}$ from the LCS of cosmonaut to the WCS. Cosmonaut motion control is realized by 24 thrusters with the equal thrust $f$, which are located on six sides of the jet pack symmetrically relative to cosmonaut's common center of mass. Each motion of cosmonaut corresponds to its own set of thrusters, which create the total thrust force $\mathbf{F}^{\prime}=\left(F_{x}^{\prime}, F_{y}^{\prime}, F_{z}^{\prime}\right)^{\mathrm{T}}$ and torque $\boldsymbol{\tau}^{\prime}=\left(\tau_{x}^{\prime}, \tau_{y}^{\prime}, \tau_{z}^{\prime}\right)^{\mathrm{T}}$ relative to the LCS of cosmonaut, where $F_{x}^{\prime}, F_{y}^{\prime}, F_{z}^{\prime} \in\{-F, 0, F\}$, $\tau_{x}^{\prime} \in\left\{-T_{x}, 0, T_{x}\right\}, \quad \tau_{y}^{\prime} \in\left\{-T_{y}, 0, T_{y}\right\}, \quad \tau_{z}^{\prime} \in\left\{-T_{z}, 0, T_{z}\right\}$. Herewith $F=4 f, T_{x}=4 f L_{x}, \quad T_{y}=4 f L_{y}$, $T_{z}=4 f L_{z}$, where $L_{x}, L_{y}$ and $L_{z}$ are the arms of thrusters relative to the cosmonaut's center of mass.

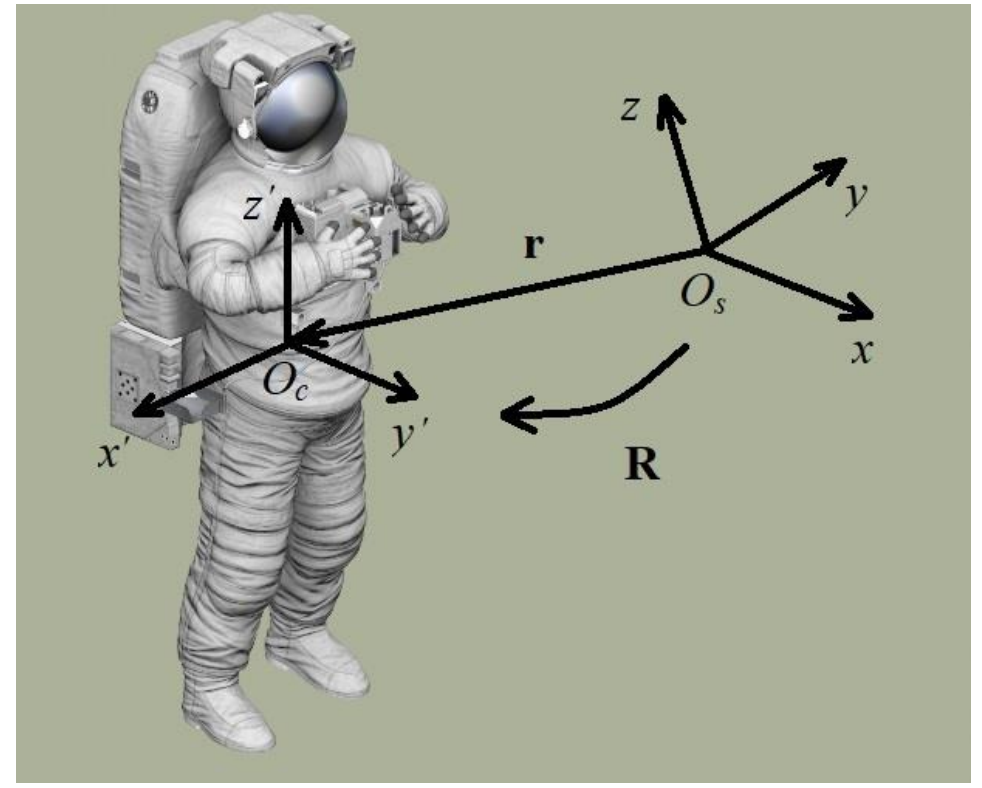

Figure 5: Virtual model of cosmonaut with jet pack

In the absence of gravity and without taking into account the influence of the ISS on cosmonaut motion, the dynamics of its linear motion is described by differential equations in the form of Newton's second law:

$$
m \dot{\mathbf{v}}=\mathbf{R F}^{\prime},
$$


where $m$ is the total mass of cosmonaut, spacesuit and jet pack, $\mathbf{v}=\left(v_{x}, v_{y}, v_{z}\right)^{\mathrm{T}}$ is the linear velocity of cosmonaut in the WCS.

The dynamics of cosmonaut's rotational motion is described by the Euler differential equations:

$$
\begin{aligned}
& I_{x} \dot{\omega}_{x}^{\prime}-\left(I_{y}-I_{z}\right) \omega_{y}^{\prime} \omega_{z}^{\prime}=\tau_{x}^{\prime} ; \\
& I_{y} \dot{\omega}_{y}^{\prime}-\left(I_{z}-I_{x}\right) \omega_{x}^{\prime} \omega_{z}^{\prime}=\tau_{y}^{\prime} ; \\
& I_{z} \dot{\omega}_{z}^{\prime}-\left(I_{x}-I_{y}\right) \omega_{x}^{\prime} \omega_{y}^{\prime}=\tau_{z}^{\prime},
\end{aligned}
$$

where $I_{x}, I_{y}$ and $I_{z}$ are the principal moments of inertia, $\boldsymbol{\omega}^{\prime}=\left(\omega_{x}^{\prime}, \omega_{y}^{\prime}, \omega_{z}^{\prime}\right)^{\mathrm{T}}$ is the angular velocity of cosmonaut in its LCS.

In that way, the dynamics for linear and rotational motion of cosmonaut is described by the equations (3) and (4), in which the components of the vectors $\mathbf{F}^{\prime}$ and $\boldsymbol{\tau}^{\prime}$ are the control variables.

Cosmonaut motion control is realized by control panel in manual and automatic modes. Deflections of the joystick in manual mode set commands to activation of thrusters, while automatic mode provides the attitude stabilization and cosmonaut movement to predetermined position. For synthesis of control actions in the VES, an approach is proposed, in which the readings of virtual sensors are used that measure position, angular velocity and orientation of cosmonaut model relative to the ISS model. The attitude stabilization of cosmonaut model along the $x^{\prime}$-axis of its LCS will be provided by relay control with feedback according to the angular velocity sensor:

$$
\tau_{x}^{\prime}=\left\{\begin{array}{ll}
-T_{x} \operatorname{sgn} \omega_{x}^{\prime}, & \left|\omega_{x}^{\prime}\right|>\varepsilon \\
0, & \left|\omega_{x}^{\prime}\right| \leq \varepsilon
\end{array} .\right.
$$

Control law (5) includes a dead zone $\left|\omega_{x}^{\prime}\right| \leq \varepsilon$, which reduces the number of switches in a neighborhood of $\omega_{x}^{\prime}=0$. Similarly, the attitude stabilization control of virtual cosmonaut model along the $y^{\prime}$ and $z^{\prime}$ axes can be obtained.

In turn, proposed solution for automatic return of cosmonaut model is to ensure the velocity towards to target. Let the target position of model be specified by the vector $\mathbf{r}_{d}=\left(x_{d}, y_{d}, z_{d}\right)^{\mathrm{T}}$. Then target motion velocity of cosmonaut model is given in the following form

$$
\mathbf{v}_{d}= \begin{cases}v_{r} \frac{r_{d}-r}{\left\|r_{d}-r\right\|}, & \left\|r_{d}-r\right\|>l ; \\ 0, & \left\|r_{d}-r\right\| \leq l,\end{cases}
$$

where $v_{r}$ is the specified value of cosmonaut velocity modulus, $l$ is the specified distance.

Linear velocity (6) provided by relay control

$$
\mathbf{F}^{\prime}=-F \operatorname{sgn}\left(\mathbf{e}_{v}^{\prime}\right)
$$

where $\mathbf{e}_{v}^{\prime}=\mathbf{R}^{-1}\left(\mathbf{v}_{d}-\mathbf{v}\right)$ is the residual vector in the LCS of cosmonaut model.

\section{Simulation results}

Methods and approaches proposed in this paper were implemented in our prototype of training complex for cosmonaut rescue. To control virtual cosmonaut model, a block diagram scheme was created. Its structure is shown in Figure 6.

Connection of training complex's hardware is implemented in the form of blocks that interconnect with hand and head tracking devices, receive from them and then send the target coordinate values (position and orientation of the operator's head and hands, as well as flexion angles of fingers) to the scheme. On the basis of obtained coordinates and virtual sensors readings (feedback in this scheme), joint torques of virtual hands model are computed according to Eq. (2). The vector $\boldsymbol{\alpha}$ of head rotation angles are used to compute the voltages $\mathbf{U}$ by means of PD controllers and then sent to electric motors of virtual camera. During the simulation, virtual hand interacts with virtual control panel, changing states of its elements (button press, joystick rotations), which are combined in the form of a vector $\mathbf{s}$. 
Further, this vector is involved in the computation of commands $\mathbf{t}$ to turn on and off thrusters, where $t_{i} \in\{0,1\}, i=\overline{1,24}$. In the automatic mode of cosmonaut model motion control, virtual sensors readings of its position and angular velocity are applied in accordance with the control laws (5) and (7).

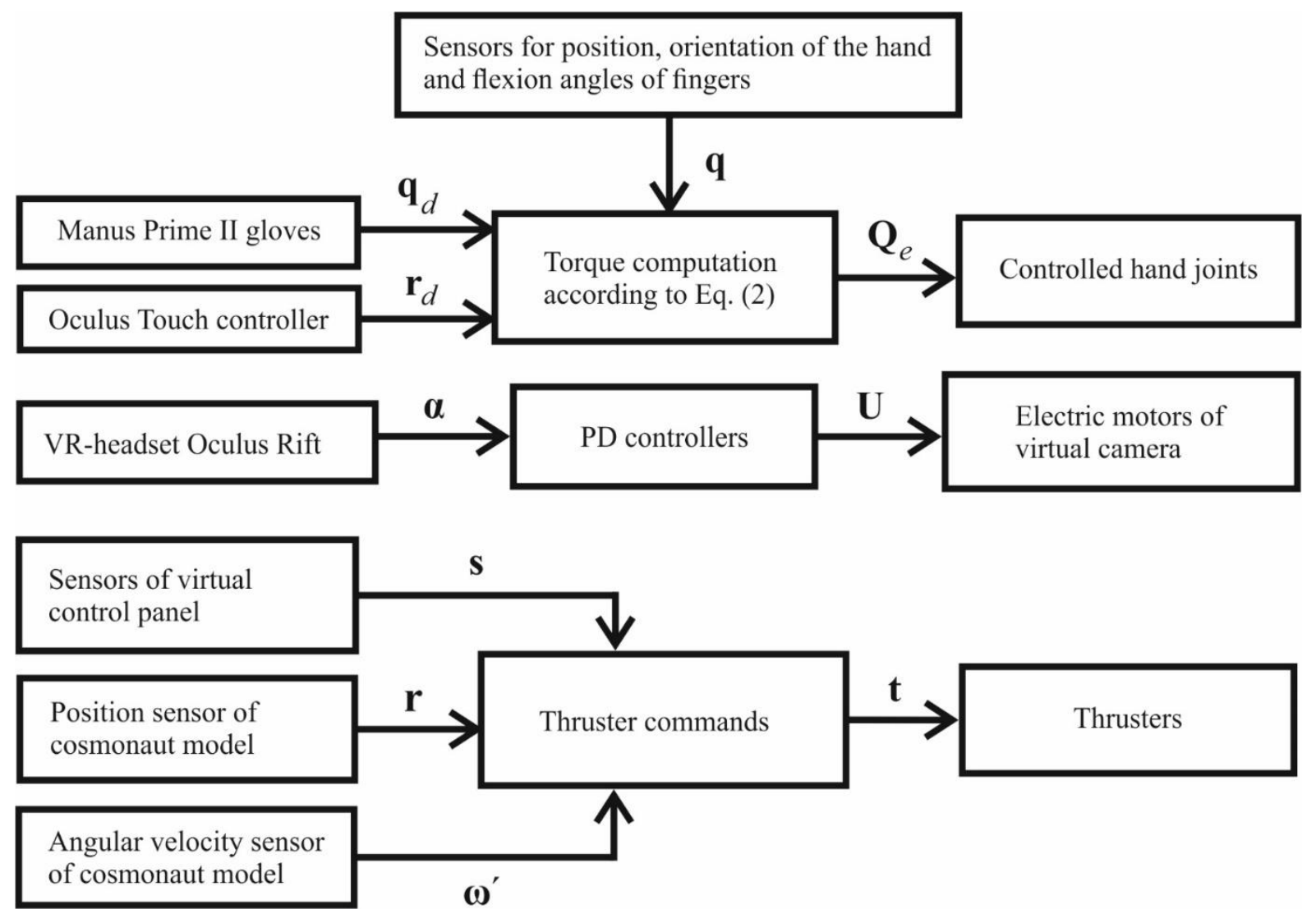

Figure 6: Structure of the control scheme

The emergency simulation in developed training complex was carried out on the example when cosmonaut separated from the ISS and has non-zero separation speeds. In this scenario, trained cosmonaut must to power virtual control panel and select automatic attitude hold mode by pressing the joystick's button. At the end of rotation, cosmonaut needs to change his attitude so that the ISS is in the center of his field of view. To do this, it is necessary to switch control "MODE" to the "ROT" position and make rotations in attitude control mode. As soon as the cosmonaut visually found the ISS, he must activate translational motion (switch control "MODE" to the "TRAN") and by deflecting the joystick to ensure his motion towards the station. Meanwhile, training assumes that fuel reserves and battery level of control panel are limited. During training, cosmonaut can enable automatic reset mode by pressing the "RTRN" button, in which he moves to the nearest predetermined position on the ISS. Figure 7 shows operator training by immersing him in virtual environment using virtual reality headset and VR gloves. Using his hands, movements of which are copied by the virtual ones, the operator controls cosmonaut model interacting with virtual panel elements.

\section{Acknowledgements}

The publication is made within the state task of Federal State Institution "Scientific Research Institute for System Analysis of the Russian Academy of Sciences" on "Carrying out basic scientific researches (47 GP)" on topic No. FNEF-2021-0012 "Virtual environment systems: technologies, methods and algorithms of mathematical modeling and visualization. 0580-2021-0012" (Reg. No. 121031300061-2). 


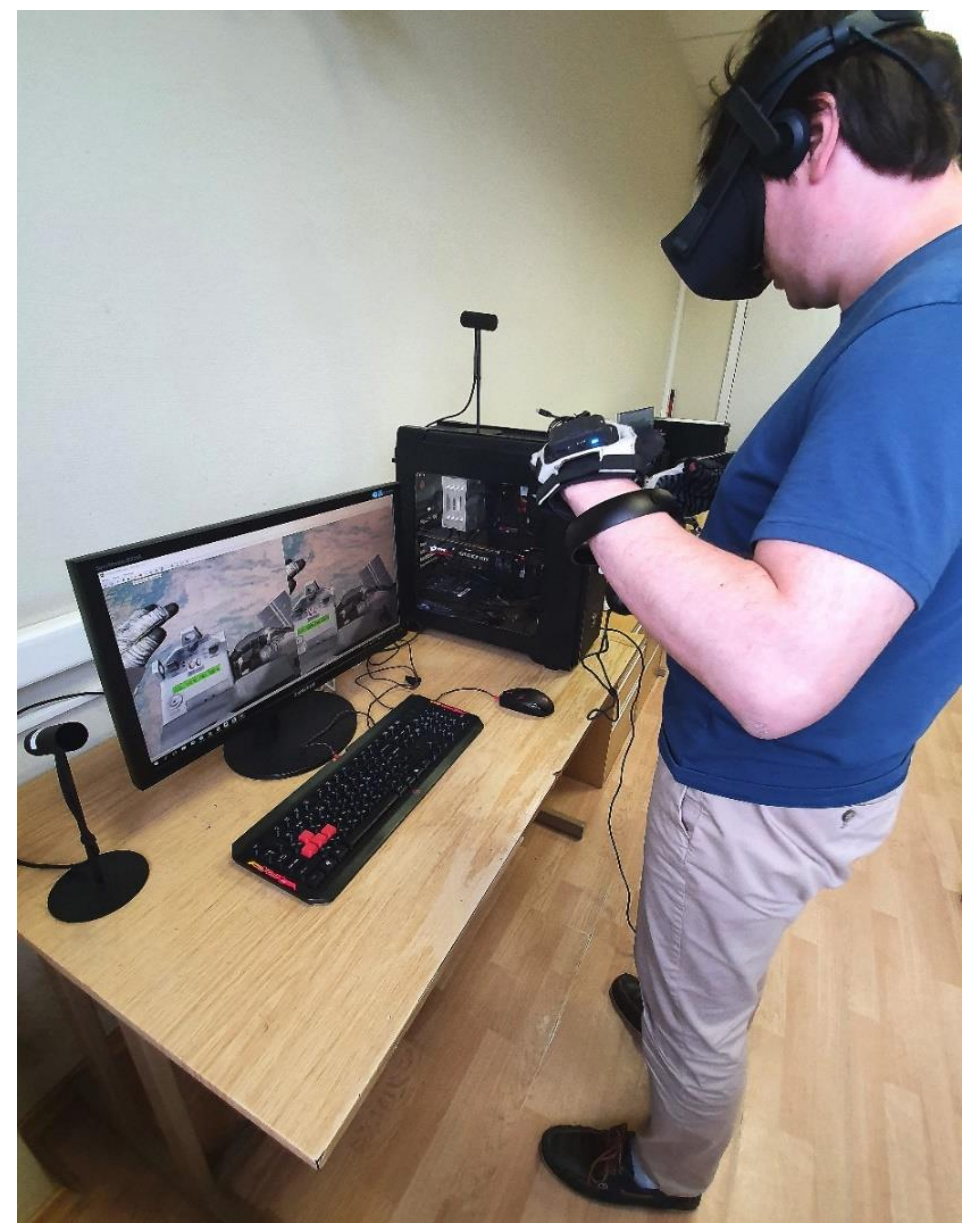

Figure 7: Operator training with virtual reality headset and VR gloves

\section{References}

[1] R. K. Fullerton, EVA Tools and Equipment Reference Book. NASA Johnson Space Center, JSC20466 Rev. B, Nov. 20, 1993.

[2] P. M. Handley, S. K. Robinson, K. R. Duda, Z. Prasov, S. P. York, and J. J. West, Real-time performance metrics for SAFER self-rescue, in: 45th International Conference on Environmental Systems, 2015, Bellevue, Washington, pp. 1-14.

[3] Yuqing Liu, Shanguang Chen, Guohua Jiang, Xiuqing Zhu, Ming An, Xuewen Chen, Bohe Zhou, and Yubin Xu, VR simulation system for EVA astronaut training, in: Proceedings of AIAA Space 2010 Conference \& Exposition, Anaheim California, 2010. doi: 10.2514/6.2010-8696.

[4] M. B. Bruguera, V. Ilk, S. Ruber, and R. Ewald, Use of virtual reality for astronaut training in future space missions - spacecraft piloting for the Lunar Orbital Platform - Gateway (LOP-G), in: 70th International Astronautics Congress, Washington D.C., 2019.

[5] A. D. Garcia, J. Schlueter, and E. Paddock, Training astronauts using hardware-in-the-loop simulations and virtual reality, in: AIAA SciTech Forum, Orlando, FL, 2020. doi: 10.2514/6.20200167.

[6] M. V. Mikhaylyuk, and M.A. Torgashev, The visual editor and calculation module of block diagrams for simulation and training complexes, Software \& Systems, 4 (2014): 10-15.

[7] A. A. Shabana, Computational dynamics, $3^{\text {rd }}$ edition, John Wiley \& Sons Ltd, 2010.

[8] M.V. Mikhaylyuk, E.V. Strashnov and P.Yu. Timokhin, Algorithms of multibody dynamics simulation using articulated-body method, Mathematica Montisnigri, 39 (2017): 133-145. 